\title{
Foreword to papers on magnetic sensitivity in birds
}

\author{
DONALD R. GRIFFIN \\ The Rockefeller University, New York, New York
}

\begin{abstract}
A magnetic sensitivity permitting orientation with respect to the earth's magnetic field would be useful to a wide variety of mobile animals. The evidence for the existence of such a sensitivity has been thoroughly reviewed by Able (1980) and in several chapters of a recent book edited by Kirschvink, Jones, and MacFadden (1985). Unfortunately, despite extensive efforts by numerous investigators using a wide array of animals, the positive evidence remains rather weak. Usually the animals show apparent effects of earth-strength magnetic fields only some of the time. This has naturally led to efforts to standardize conditions and isolate critical variables in the hope that unambiguous results not only would demonstrate the existence of sensitivity to the earth's field but would permit analysis of the physiological mechanisms that underlie it. The following papers report such experiments, involving a variety of procedures, all of which yielded negative results. Many other experiments of this general kind have led to negative results, which are not ordinarily published. But the problem of magnetic sensitivity is of such general interest, and negative results of experiments of this sort are so common, that publication of detailed procedures and results is warranted in order to balance the published record and aid in the design of future experiments.
\end{abstract}

The physics of the earth's magnetic field has been reviewed by Skiles (1985), but a brief and elementary summary of terrestrial magnetism and the units in which it is measured may be helpful to many readers. The most familiar effect of the earth's field is the orientation of a magnetic compass needle, one pole of which points toward the north magnetic pole of the earth. Ordinary compasses are constructed so that the needle responds only to the horizontal component of the earth's field, but if the needle is free to tip up or down it shows the vertical component of inclination. Close to the magnetic equator, roughly halfway between the north and south magnetic poles, the vertical component is effectively zero, and near the magnetic poles the horizontal component is very weak. Although the horizontal component is presumably the most useful for orientation, the actual physical stimulus is the earth's total field, which, in the temperate latitudes where most experiments have been conducted, points downward at angles on the order of $50^{\circ}$ to $75^{\circ}$.
The earth's field might be detected by a mechanical sensitivity to the forces exerted on a magnetized object comparable to the familiar compass needle. But magnetic fields also have an important interaction with electric currents, which affords other possibilities for biological sensitivity. When a conductor moves through the earth's magnetic field, a small electrical voltage is induced across it, the magnitude depending on the speed of movement and the strength of the field. The same effect is produced by changing the strength of the magnetic field, so that experimentally generated fields can reproduce the electrical induction caused by motion through the earth's field by changing the intensity of the field. Linear changes of intensity simulate the magnetic induction caused by linear motion at constant speed.

Bar magnets or other solid pieces of magnetized material generate artificial fields and have been used to test for magnetic sensitivity in animals, but much more uniform fields can easily be produced by taking advantage of the fact that when an electric current flows through a conductor such as a metal wire, it generates a magnetic field in the immediate vicinity. A current through a circular coil produces a field perpendicular to the plane of the coil; a current through two identical coils separated by their radius (Helmholtz coils) produces a roughly constant field; and a current through a simple arrangement of square coils, described by Rubens (1945), produces a more nearly uniform field. These two types of coils have been widely used in experiments designed to detect magnetic sensitivity in animals. Direct currents through such coils produce constant magnetic fields, and varying currents produce magnetic fields that follow the waveform of the electric current.

The strength of the earth's magnetic field is often expressed in gauss (G) or oersteds, nearly identical units. But recently the tesla, equal to $10,000 \mathrm{G}$, has come into increasing use. The total intensity of the earth's field varies geographically between about 0.25 and $0.65 \mathrm{G}$, and in temperate latitudes the horizontal component is on the order of $0.25 \mathrm{G}$. The earth's field varies in time, with short-term variations in magnetic storms commonly on the order of a few hundred gamma, where 1 gamma = $1 / 100,000 \mathrm{G}$. The earth's field also varies over short distances due to deposits of iron, and these variations are 
also on the order of $1 / 1,000$ of the earth's field. Magnetic storms and magnetic anomalies have been reported to affect the orientation of birds, as reviewed by Able (1980), Gould (1985), and Keeton (1974). These reported effects imply sensitivity not only to the earth's overall field of roughly $0.5 \mathrm{G}$ but to variations over time and space of less than $0.1 \%$.

The influential paper by Keeton (1971) is typical of the frustrating picture presented by experiments on magnetic sensitivity. He reported differences in homeward orientation of pigeons depending on whether they carried bar magnets or brass weights of comparable size and weight. Unfortunately, these effects were variable, both in the experiments reported by Keeton and, apparently, in subsequent replications. There was sometimes no difference between experimental and control pigeons, but in all cases in which there was a significant difference, the birds with magnets were less well oriented. How can one deal with experimental evidence of this kind? Perhaps there are important variables affecting the orientation of homing pigeons and other birds that have not yet been identified and therefore cannot be controlled experimentally. The positive evidence is certainly suggestive, and the whole subject calls for more thorough and critical investigation. Let us hope that the following papers will stimulate the design of new approaches that may finally clarify this puzzling situation.

\section{REFERENCES}

ABLE, K. P. (1980). Mechanisms of orientation, navigation and homing. In S. A. Gauthreaux, Ir. (Ed.), Animal migration, orientation, and homing. New York: Academic Press.

GouLD, J. L. (1985). Are animal maps magnetic? In J. L. Kirschvink, D. S. Jones, \& B. J. MacFadden (Eds.), Magnetite biomineralization and magnetoreception in organisms, a new biomagnetism (chap. 12, pp. 257-268). New York: Plenum Press.

KeEton, W. T. (1971). Magnets interfere with pigeon homing. Proceed ings of the National Academy of Sciences, 68, 101-106.

KEETON, W. T. (1974). The orientational and navigational basis of homing in birds. In D. S. Lehrman, J. S. Rosenblatt, R. A. Hinde, \& E. Shaw (Eds.), Advances in the study of behavior (Vol. 5, pp. 47-132). New York: Academic Press.

KIRSCh Vink, J. L., Jones, D. S., \& MACFAdDEN, F. J. (Eds.) (1985). Magnetite biomineralization and magnetoreception in organisms, a new biomagnetism. New York: Plenum Press.

RuBENS, S. M. (1945). Cube surface coil for producing uniform magnetic fields. Review of Scientific Instruments, 16, 243-245.

SKILES, D. D. (1985). The geomagnetic field: Its nature, history, and biological relevance. In J. L. Kirschvink, D. S. Jones, \& B. J. MacFadden (Eds.), Magnetite biomineralization and magnetoreception in organisms, a new biomagnetism (chap. 3, pp. 43-102). New York: Plenum Press. 